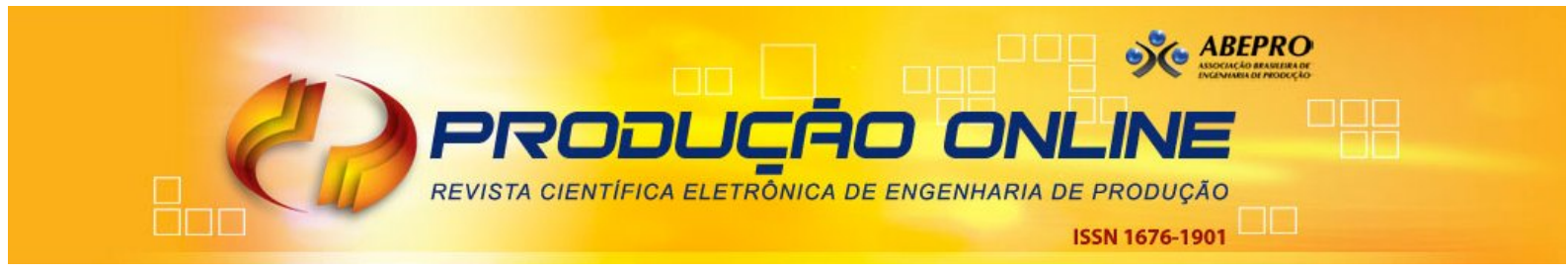

\title{
PROJETO DE SERVIÇO: UMA ANÁLISE DOS MÉTODOS E TÉCNICAS EXISTENTES SOB A ÓTICA DA ÁREA DE GESTÃO DE OPERAÇÕES DE SERVIÇOS
}

\section{SERVICE DESIGN: A REVIEW OF METHODS AND TECHNIQUES INTHE POINT OF VIEW OF SERVICE OPERATIONS MANAGEMENT AREA}

\author{
Noel Torres Júnior* E-mail: noel@face.ufmg.br \\ Nayara de Oliveira Gonzaga* E-mail: nayara1 74@hotmail.com \\ *Universidade Federal de Minas Gerais (UFMG), Belo Horizonte, MG
}

Resumo: O setor de serviços no Brasil, assim como em vários países desenvolvidos, é responsável pela geração de renda e emprego para uma parcela significativa da população. Decorrência dessa relevância econômica, as organizações de serviços se deparam com um mercado cada vez mais exigente e competitivo, que demanda maior capacidade de inovação e planejamento. A despeito disto, o processo de desenvolvimento de serviços permanece como um tema pouco explorado na literatura. Vários aspectos concernentes ao seu planejamento e gerenciamento continuam sem concepções ou proposições claras que orientem o seu adequado tratamento. Fruto desta situação, os métodos e as técnicas que auxiliem o projeto dos serviços não estão devidamente disseminados e ainda se encontram dispersos na literatura de gerenciamento de operações de serviços. Diante deste quadro, o presente trabalho, à luz de uma revisão da literatura, e tendo como referência os elementos que compõe o projeto de serviço segundo os trabalhos de Machuca, González-Zamora e AguilarEscobar (2007) e Edvardsson (1997) aponta e analisa quais métodos e técnicas podem ser utilizados nesta atividade. Verificou-se que o conhecimento acerca dos métodos e técnicas se encontra difuso na literatura e o mesmo se concentra no projeto do processo e na definição da localização das instalações dos serviços.

Palavras-Chave: Métodos e Técnicas. Projeto de Serviços. Desenvolvimento de Serviços.

Abstract: As in many developing countries, the service sector in Brazil is responsible for generating income and employment for a significant portion of the population. Due to this economic relevance, service organizations are faced with an increasingly demanding and competitive market. This demand greater innovation and business planning. Despite this, the process of service development remains a relatively unexplored subject in the literature. Several issues regarding the planning and management remain without clear ideas or propositions that guide their appropriate treatment. The result of this situation, the methods and techniques that help design the services are not properly disseminated. These are scattered through the literature of operations management services. Given this framework, the light of a review of the literature and concerning the elements that compose the service project according to the works of Machuca, Zamora-Gonzalez and Aguilar-Escobar (2007) and Edvardsson (1997), the present work points and examine the methods and techniques suggested by many authors and works on the area of Service Operations. It was found that the knowledge about the methods and techniques is diffuse. This knowledge is concentrated on the design process and determining the location of services.

Keywords: Methods and Techniques. Project Services. Development Services 


\section{INTRODUÇÃO}

O setor de serviços no Brasil, assim como em vários países desenvolvidos, é responsável pela geração de renda e emprego numa parcela significativa da população (LOVELOCK, WIRTZ e HEMZO, 2011). Decorrência dessa relevância econômica, as organizações de serviços se deparam com um mercado cada vez mais exigente e competitivo que demanda maior capacidade de inovação e planejamento (FITZSIMMONS e FITZSIMMONS, 2000; CHESBROUGH e DAVIES, 2010). Além disso, a fronteira entre bens e serviços tem se tornado cada vez mais tênue, visto que os bens tendem a incorporar serviços associados aos seus produtos centrais como uma estratégia de diferenciação (OLIVA e KALLENBERG, 2003). A despeito destes fatos, o projeto de serviços $^{1}$ ainda não é tema devidamente explorado pela literatura da área de operações. Uma comparação do desenvolvimento de produtos com o desenvolvimento de serviços revela que o último ainda não está consolidado (MENOR, TATIKONDA e SAMPSON, 2002). Como uma consequência desta situação, os autores Zeithaml, Bitner e Gremler (2011) comentam que, em grande parte das vezes, os serviços surgem e são implementados com base em opiniões individuais dos funcionários que estão envolvidos no processo, fazendo com que este ocorra de modo predominantemente subjetivo e pouco estruturado. Além disso, uma análise dos principais livros didáticos de gerenciamento de operações de serviços revela que os métodos e as técnicas que auxiliam no projeto de serviços não são numerosos e se apresentam de maneira dispersa e desintegrada. Isto por sua vez, não favorece o maior desenvolvimento e a divulgação de boas práticas para o projeto de serviços nas organizações.

Diante deste quadro, o presente trabalho, à luz de uma revisão da literatura na área de Operações de Serviços e tendo como referência os elementos que compõe o projeto de serviço segundo os trabalhos de Machuca, González-Zamora e Aguilar-Escobar (2007) e Edvardsson (1997) aponta e analisa quais métodos e técnicas podem ser utilizados nesta atividade. Para tanto, inicialmente, o trabalho apresenta o método de pesquisa utilizado. Posteriormente, mostra os principais elementos que devem ser desenvolvidos no projeto dos serviços. Para cada um deles, o trabalho indica quais métodos e técnicas foram sugeridas pelos diversos

\footnotetext{
${ }^{1}$ Este trabalho utiliza o termo Projeto de Serviços como sinônimo de Desenvolvimento de Serviços.
} 
autores e trabalhos da área. Finalmente, algumas considerações são tecidas sobre o tema proposto e as limitações do trabalho são também elencadas.

\section{MÉTODO DE PESQUISA}

O trabalho pode ser entendido como sendo de natureza exploratória e qualitativa (GIL, 1991), uma vez que irá proporcionar uma visão geral do desenvolvimento do projeto de serviços, de modo a propiciar maior conhecimento acerca dos seus componentes, dos métodos ou técnicas que auxiliam no projeto dos serviços.

Para a realização deste trabalho foi necessário uma melhor compreensão de quais elementos compõe o projeto dos serviços. Para tanto, os autores consultaram diversos textos diretamente relacionados ao tema. Como resultado desta atividade, os autores escolheram os elementos do projeto de serviço baseando-se nos trabalhos de Machuca, González-Zamora e Aguilar-Escobar (2007) e Edvardsson (1997). Estes são expostos a seguir:

- Abordagem: o projeto do serviço pode ser feito segundo alguma abordagem genérica. Em um extremo, pode-se conceber um serviço por meio de uma abordagem tipicamente industrial do tipo "linha de produção" (LEVITT, 1976). Em outro extremo, o serviço pode ser concebido de maneira que o cliente tenha uma grande participação e interação com o processo de prestação. Dentro destes dois extremos, várias abordagens intermediarias podem ser definidas (CHASE, 1978 e 2011);

- Produto: o projeto deverá definir qual o produto (bens e serviços) que deverá ser entregue aos clientes. Esta definição será feita mediante a explicitação de dois elementos: i) Conceito - refere-se aos benefícios e utilidades que o serviço, como um todo, e seus subserviços pretendem fornecer ao cliente; ii) Bens Facilitadores - correspondem aos materiais adquiridos ou consumidos pelos compradores, ou ainda, aos itens fornecidos pelos clientes necessários à realização dos serviços; 
- Processo: o projeto deverá definir como o produto será entregue. Esta definição será feita mediante a explicitação de dois elementos: i) Etapas e atividades que compõem o processo. Estas podem ser entendidas como a rede de atividades paralelas e/ou sequenciais que devem ser realizadas para consecução do serviço; ii) Capacidade e duração destas etapas e atividades;

- Sistema: existe para dar suporte e funcionamento ao processo de serviço. Este pode ser desdobrado em quatro sub-elementos: i) Recursos Técnicos Físicos - incluem os recursos da empresa, dos fornecedores e clientes que são necessários para o serviço. As evidências físicas dos serviços ou Cenário de Serviços devem ser planejadas neste elemento (BITNER, 1992); ii) Sistemas de Controle - incluem vários elementos, tais como a estrutura organizacional a ser adotada, o sistema de suporte administrativo (exemplos: sistema financeiro, sistema de pagamento, sistema de informação, sistema de padrões), a interação, o diálogo com os clientes (exemplos: sistemas de feedback e controle a respeito das reclamações, da insatisfação. Isto incluí os horários de funcionamento, número do telefone, etc.). Os sistemas de controle possibilitam o gerenciamento das atividades rotineiras da organização; iii) Empregados - refere-se ao projeto do posto de trabalho de cada empregado; iv) Clientes - estes devem ser entendidos como parte do recurso da empresa, portanto, neste aspecto, o sistema de serviço deve ser planejado para que o cliente interaja e participe do processo de serviço da melhor maneira possível. O papel, conhecimento e habilidades necessários para a sua adequada participação também devem ser planejados neste sub-elemento.

Uma vez que estes elementos foram identificados e definidos, iniciou-se o levantamento bibliográfico acerca dos métodos e das técnicas. Estes podem ser entendidos como conhecimentos estruturados em passos ou etapas que buscam atingir um determinado objetivo e não são tão abrangentes como os conceitos ou filosofias. No projeto de bens manufaturados, existem vários exemplos de métodos e 
técnicas, tais como FTA (Fault Tree Analysis), FMEA(Failure Modes, Effects and Criticality Analysis), FAST (Function Analysis Sytem Technique), etc. (ROZENFELD et al., 2006).

A revisão bibliográfica foi feita considerando dois tipos de publicações. Inicialmente procurou-se conhecer como o tema abordado é trabalhado nos livros textos de gerenciamento de operações de serviços. Para este fim, os autores consultaram os sites de duas grandes livrarias virtuais (Amazon e Livraria Cultura). Posteriormente, os autores procuraram artigos científicos sobre este tema. Com este propósito, o Portal Capes, a base de dados EBSCO Business Premier e Scielo foram consultados. Vários periódicos da área de operações foram examinados. Os seguintes periódicos foram consultados: Harvard Business Review(HBR); International Journal of Operations and Production Management(IJOPM); International Journal of Service Industry Management (IJSIM); Journal of Operations Management (JOM); Journal of Service Research (JSR); Production and Operations Management (POM); Revista Produção Online; Revista Produção; Service Bussiness (SB) e The Service Industries Journal (SIJ).

A busca por livros de gerenciamento de operações de serviços da língua portuguesa e inglesa revelou a existência de 16 títulos. Estes são apresentados no Quadro 1. 
Quadro 1 - Livros de gerenciamento de operações de serviços encontrados na Pesquisa

\begin{tabular}{|l|c|}
\hline \multicolumn{1}{|c|}{ Livros } & Ano \\
\hline $\begin{array}{l}\text { Sasser, W. E.; Olsen, R. P.; Wyckoff, D. D. Management of service } \\
\text { Operations: Text, Cases, and Readings, Boston: Allyn and Bacon Inc. }\end{array}$ & 1978 \\
\hline $\begin{array}{l}\text { Voss, C.; et al. Operations Management in Service Industries and the } \\
\text { Public Sector, John Wiley \& Sons Ltd. }\end{array}$ & 1990 \\
\hline $\begin{array}{l}\text { Normann, R. Administração de serviços: estratégia e liderança na } \\
\text { empresa de serviços, São Paulo: Editora Atlas. }\end{array}$ & 1993 \\
\hline $\begin{array}{l}\text { Gianesi, I. G. N.;Corrêa, H. L. Administração estratégica de serviços, } \\
\text { São Paulo: Editora Atlas. }\end{array}$ & 1994 \\
\hline $\begin{array}{l}\text { Ramaswamy, R. Design and Management of Service Process: } \\
\text { keeping customer for life. Prentice Hall. }\end{array}$ & 1996 \\
\hline $\begin{array}{l}\text { Schmenner, R. W. Administração de Operações em Serviços, São } \\
\text { Paulo: Editora Futura. }\end{array}$ & 1999 \\
\hline $\begin{array}{l}\text { Téboul, J. A era dos serviços: uma nova abordagem de } \\
\text { gerenciamento, Rio de Janeiro: Qualitymark. }\end{array}$ & 1999 \\
\hline $\begin{array}{l}\text { Haksever, C.;et al. Service Management and Operations, New } \\
\text { Jersey: Prentice Hall. }\end{array}$ & 2000 \\
\hline $\begin{array}{l}\text { Corrêa, H. L.; CAON, M. Gestão de serviços: lucratividade por meio } \\
\text { de operações e de satisfação dos clientes, São Paulo: Atlas. }\end{array}$ & 2002 \\
\hline $\begin{array}{l}\text { Metters, R.; King-Metters, K.; Pullman, M. Successful Service } \\
\text { Operations Management, Thomson South-Western. }\end{array}$ & 2003 \\
\hline $\begin{array}{l}\text { Johnston, R.; Clark, G. Service Operations Management: improving } \\
\text { service delivery, Pearson Education Limited. }\end{array}$ & 2008 \\
\hline $\begin{array}{l}\text { Teboul, J. Serviços em Cena: o diferencial que agrega valor ao seu } \\
\text { negócio, Brasília: IEL/NC. }\end{array}$ & 2008 \\
\hline $\begin{array}{l}\text { Grönroos, C. Marketing: gerenciamento e serviços, Rio de Janeiro: } \\
\text { Elsevier. }\end{array}$ & 2010 \\
\hline $\begin{array}{l}\text { Fitzsimmons, J. A; Fitzsimmons, M.J. Administração de Serviços: } \\
\text { operações, estratégia e tecnologia de informação, Porto Alegre: } \\
\text { Bookman Companhia Editora. }\end{array}$ & 2011 \\
\hline $\begin{array}{l}\text { Mello, C. H. P. et al. Gestão do Processo de Desenvolvimento de } \\
\text { Serviços. São Paulo: Atlas. }\end{array}$ & 2010 \\
\hline $\begin{array}{l}\text { Lovelock, C.; Wirtz, J.; Hemzo, M. A. Marketing de Serviços: pessoas, } \\
\text { tecnologia e estratégia, São Paulo: Pearson Prentice Hall, 70 Edição. }\end{array}$ & 2009 \\
\hline
\end{tabular}

Dos livros consultados e apresentados no Quadro 1, considerando o ano de edição e seu conteúdo, os autores selecionaram 11 deles: Corrêa e Caon (2002); Fitzsimmons e Fitzsimmons (2010); Grönroos (2008); Haksever et al (2000); Johnston e Clark (2008); Lovelock, Wirtz e Hemzo (2011); Mello et al (2010); Metters, King-Metters e Pullman (2003); Ramaswamy (1996); Schmenner (1999); e Teboul (2008). 
Logo em seguida, procurou-se nas bases de dados os artigos relacionados aos métodos/técnicas descritos nos livros consultados. Esta busca baseou-se no próprio nome do método/técnica ou na referência bibliográfica existente sobre o mesmo. A consulta a estes artigos auxiliou os autores na compreensão do método/técnica e facilitou a atividade de enquadramento do mesmo no Framework dos elementos do Projeto de Serviço proposto no trabalho.

A análise dos textos proporcionou traçar um Quadro Geral dos métodos e das técnicas para o Projeto de Serviços. Este é apresentado em cada um dos quatros elementos do Projeto de Serviço presentes nas Figuras 1, 2 e 3. Estes são discutidos na Seção 03 deste trabalho.

\section{O PROJETO DE SERVIÇOS NA VISÃO DE DIVERSOS AUTORES}

\subsection{Abordagens de Serviços}

Os autores Fitzsimmons e Fitzsimmons (2010) propõem quatro abordagens genéricas para o projeto de serviços. A primeira delas é a abordagem baseada na lógica da linha de produção industrial. Esta consiste numa adaptação da operação de serviços aos princípios que orientam o funcionamento da linha de produção industrial. Neste sentido, a operação de serviços adota várias das práticas existentes no sistema industrial desse tipo. São exemplos delas: imposição de limites de discrição e arbítrio aos funcionários; forte divisão do trabalho; substituição de pessoas por tecnologia, sempre que possível; e padronização das atividades. Esta abordagem busca resultados principalmente em termos de aumento de eficiência e redução de custos. Uma segunda abordagem consiste em colocar o cliente no papel de co-produtor, incentivando a participação ativa do mesmo nas várias fases existentes no processo de entrega dos serviços. Uma terceira abordagem apresentase como intermediaria entre as duas anteriores. Esta divide o serviço em operações de alto e baixo contato, permitindo que as operações de baixo contato sejam projetadas com um enfoque técnico voltado para eficiência e as operações de alto contato sejam projetadas pela lógica de maior customização e qualidade dos serviços (CHASE, 1978 e 2010). Finalmente, uma quarta abordagem voltada para a delegação de poder pela informação. Consiste no uso intenso da Tecnologia da 
Informação nos processos, possibilitando maior delegação de poder aos empregados e clientes.

\subsection{Produto dos Serviços}

Segundo os autores Johnston e Clark (2008) o conceito de serviço pode ser entendido como a proposição do negócio. Do ponto de vista do cliente, ele é o modo pelo qual o cliente percebe os serviços da organização. Pode ainda ser considerado como o pacote de benefícios para o cliente, isto é, o que de fato agrega valor a ele. Além de ajudar a definir e entender a natureza dos serviços, o conceito pode ser usado como uma ferramenta estratégica. Dessa Maneira, os autores propõem uma forma para definição do mesmo pelas diversas áreas da organização (CLARK, JOHNSTON e SHULVER, 2000). Corrêa e Caon (2002) definem o conceito do serviço como uma ferramenta importante na unificação das visões das pessoas envolvidas na tomada de decisão dentro da organização acerca do serviço entregue. Para Mello et al. (2010) a definição do conceito de serviço surge da necessidade da organização definir as percepções que se desejam gerar nas mentes dos clientes acerca do serviço que será entregue. Entretanto, em muitos serviços, as empresas definam o seu conceito por meio de uma simples declaração sucinta. Isto por sua vez, impossibilita a correta compreensão do mesmo pelos funcionários e clientes da organização.

Ramaswamy (1996) expõe o método Pugh adaptado para a avaliação e seleção de conceitos de serviço. Uma compreensão qualitativa e clara dos conceitos do serviço é necessária ao sucesso de sua aplicação. Uma matriz de avaliação é usada para comparar os conceitos propostos. Os critérios de avaliação compõem as linhas da matriz e os conceitos são comparados por alguns critérios de performance e custos.

Os autores Lovelock, Wirtz e Hemzo (2011) propõem o Mapa de Posicionamento como ferramenta para visualizar o posicionamento competitivo, identificar desdobramentos ao longo do tempo e desenvolver cenários de potenciais respostas da concorrência para os serviços.

Teboul (2008) propõe uma matriz que possibilita a empresa especificar e identificar qual o valor que o serviço busca atender a empresa, seus clientes e 
colaboradores. Esta matriz apresenta duas dimensões: uma refere-se aos principais elementos ofertados pelo serviço e a outra refere-se aos resultados desejados para a empresa, clientes e colaboradores.

De acordo com Fitzsimmons e Fitzsimmons (2010), os bens facilitadores correspondem aos materiais adquiridos ou consumidos pelos clientes, ou ainda aos itens (bens) fornecidos pelos mesmos. A importância dos bens facilitadores no pacote de serviços depende predominância de elementos intangíveis nos serviços. Dessa forma, pode-se dizer que os bens facilitadores são os recursos necessários à realização dos serviços, e que podem ser avaliados segundo sua consistência, quantidade e seleção. Já para Corrêa e Caon (2002) os bens facilitadores são também conhecidos como elementos estocáveis e fazem parte do "pacote de valor" que as empresas entregam aos seus clientes. Podem ser divididos em dois tipos: aquele cuja propriedade é transferida durante a prestação de serviço, como por exemplo, a refeição servida numa companhia aérea; e aquele cuja propriedade não é transferida durante a prestação de serviço, por exemplo, a fita de vídeo de uma locadora, os quartos e instalações dos hotéis.

A Figura 1 mostra os métodos e as técnicas referenciados nesta seção e a fonte bibliográfica que contém tal método.

Figura 1 - Relação de métodos e técnicas para o Projeto do Produto

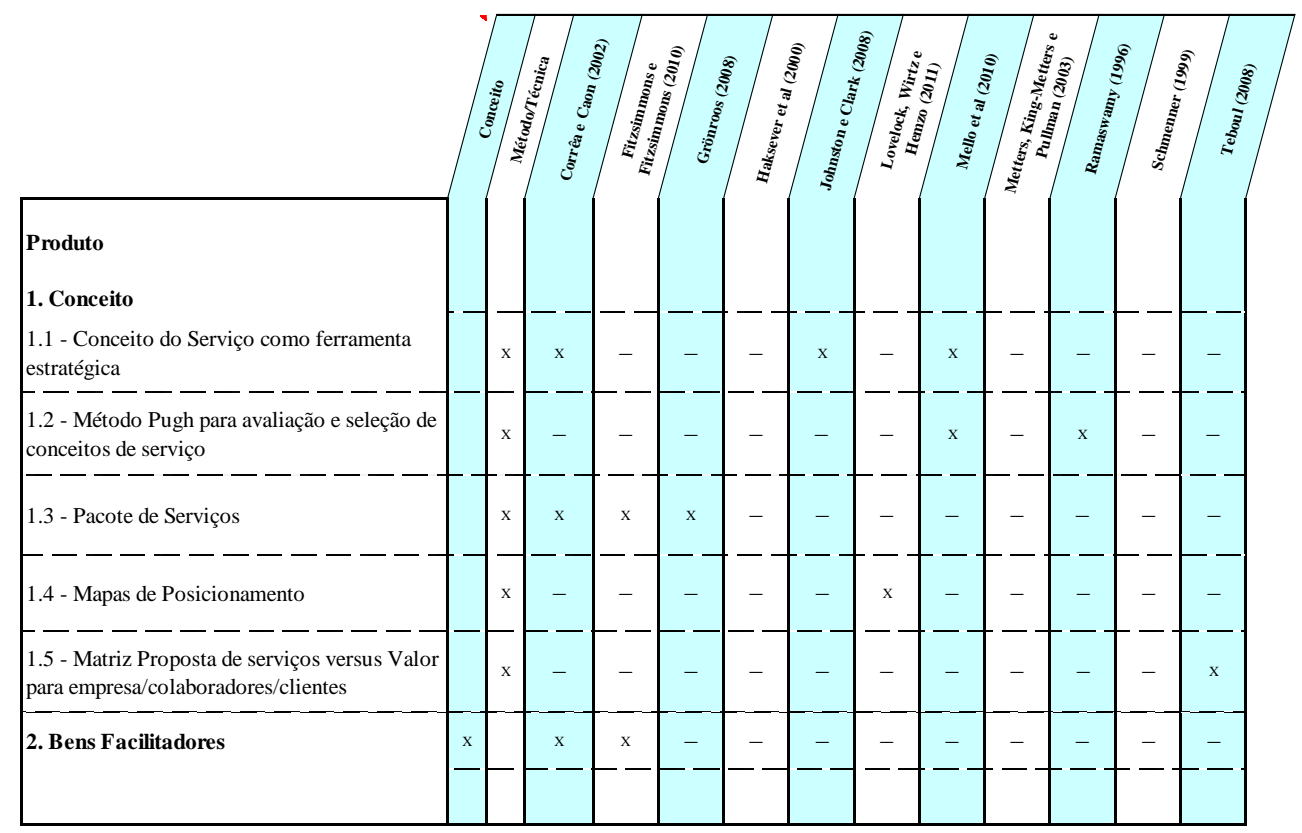

Fonte: Pesquisa dos Autores (2012). 


\subsection{Processo do Serviço}

O projeto do processo de serviço concentra um grande número de métodos ou técnicas, o mesmo está presente em todas as obras pesquisadas. O blueprinting do serviço é um método bastante referenciado nestas obras. Este consiste num mapa que descreve as principais transações existentes no processo. O mapa mostra de maneira destacada as evidências físicas percebidas pelos clientes e as atividades realizadas mediante interação dos clientes com os funcionários da linha de frente. Neste diagrama, as atividades de retaguarda são localizadas em uma parte específica do mapa. Auxilia no planejamento do serviço, uma vez que ajuda na identificação de pontos de possíveis falhas (SHOSTACK, 1984; FITZSIMMONS E FITZSIMMONS, 2010). Mello et al (2010) colocam que o service blueprint foi a primeira técnica desenvolvida especialmente para mapear os seus processos, diferenciando-se dos fluxogramas de processo por incluir novas características. Baseando-se na técnica blueprinting, os autores Corrêa e Caon (2002) criaram o Fluxo do Processo de Serviço. Além dos elementos existentes no blueprinting, este também mostra os recursos críticos e os critérios de desempenho para cada atividade. Desse modo, o Fluxo do Processo de Serviço é uma ferramenta útil na análise de processos em serviços, uma vez que é possível analisar quais atividades ou processos têm maior impacto nos momentos de contato considerados mais relevantes para o cliente.

Outro método bastante conhecido na manufatura para a representação das etapas ou atividades do processo é o fluxograma. Este é um recurso visual para a representação de um processo, em que há uma simbologia própria para sua construção. Os fluxogramas de processo descrevem sequencialmente um processo, destacando quais fases operacionais são executadas antes de outras e quais podem ser feitas em paralelo (SCHMENNER, 1999). Nesta ferramenta, os atrasos ou esperas, atividades de inspeção, operação e transporte podem ser identificados. Ele também auxilia no reconhecimento de pontos problemáticos ou pontos passíveis de intervenção para solução de problemas relacionados à qualidade (FITZSIMMONS E FITZSIMMONS, 2010).

O Poka-Yoke (dispositivo a prova de erros) é apresentado por diversos autores como um mecanismo de controle da qualidade. Eles impedem que um erro 
inevitável se torne um defeito de serviço e seja percebido pelo cliente. Podem ser baratos e simples, podendo ser usados para evitar que tanto funcionários quanto clientes cometam falhas. Podem ser agrupados em dois tipos: poka-yokes do prestador de serviço, referentes à tarefa em si, ao tratamento dado ao cliente ou aos elementos tangíveis envolvidos; e poka-yokes do cliente referentes às atividades realizadas pelos próprios clientes (CHASE e STEWART, 1994; CORRÊA E CAON, 2002; JOHNSTON e CLARK 2008; FITZSIMMONS e FITZSIMMONS, 2010).

A técnica conhecida como QFD (Quality Function Deployment) ou Desdobramento da Função Qualidade é uma abordagem sistemática para incorporar a qualidade em todas as atividades existentes no ciclo de projeto do produto. Esta técnica utiliza uma série de matrizes interconectadas que identificam as relações entres as diversas fases do desenvolvimento. A Casa da Qualidade, usada para traduzir as necessidades dos clientes em características do projeto do serviço, é referenciada por diversos autores consultados (RAMASWAMY, 1996; FISHER e SCHUTTA, 2003).

A análise da transação de serviço (STA - Service Transaction Analysis) pode ser entendida como uma auditoria que avalia o processo do ponto de vista do cliente. Quatro elementos críticos são julgados: o conceito do serviço, o processo do serviço, a qualidade de cada transação e a interpretação do serviço pelo cliente. A STA combina estes elementos para possibilitar uma avaliação e uma eventual melhora na experiência do cliente em relação a um processo de serviço. É um método bastante simples e eficaz, podendo ser aplicado facilmente pelos gerentes para melhorar a consciência dos funcionários acerca da experiência vivenciada pelo cliente no processo (JOHNSTON, 1999; JOHNSTON E CLARK, 2008; SANTOS e VARVASKIS, 2001).

O mapa do serviço é uma técnica derivada do service blueprint envolvendo a esquematização do serviço como um todo. O diagrama descreve o serviço desde a captação do cliente até a finalização do processo de entrega do mesmo. (KINGMANBRUNDAGE, GEORGE e BOWEN,1995; MELLO et al, 2010).

A família de técnicas IDEF (Definição de Manufatura Auxiliada por Integração com Computador) foi desenvolvida pela Força Aérea Americana para auxiliá-la no processo de desenvolvimento e avaliação de seus fornecedores. Esta é utilizada na representação de processos de serviços e as técnicas IDEFO e a IDEF3 são as mais 
utilizadas. A IDEFO é uma abordagem hierárquica ao mapeamento de processo, na qual uma descrição básica e simples do mesmo é decomposta, passo a passo, em suas atividades constituintes, para qualquer nível de detalhe desejado. A IDEFO representa as atividades, entradas, saídas, controles e recursos existentes no processo. A IDEF3 é uma técnica na qual o especialista expressa seu conhecimento a respeito de um processo particular, descrevendo o que um sistema realiza por meio da combinação de gráficos e textos que descrevem o fluxo do processo e da rede de transição do estado do objeto (MELLO et al, 2010).

O SADT (Strutured Analysis Design Technique) é uma técnica de mapeamento dos processos semelhante à IDEF0. É principalmente utilizada para mapear as operações de retaguarda. Consiste em transformar o mapeamento dos processos em uma ferramenta analítica, por meio de algumas perguntas-chave sobre o mesmo que devem ser respondidas pelo analista (MELLO et al, 2010).

A walk through audit (WTA) é uma forma de auditoria do serviço, composta por uma série de questões relativas ao processo e ao pacote de serviços oferecido pela empresa dirigidas aos clientes e gerentes do negócio. Procura analisar o serviço sob o ponto de vista do cliente (SANTOS E VARVASKIS, 2001).

A ferramenta Emotion Mapping busca obter uma visão orientada ao cliente no processo. Nesta direção, procura-se entender quais emoções positivas e negativas o cliente pode apresentar em cada uma das etapas mapeadas (JOHNSTON E CLARK, 2008).

A simulação computacional fornece uma espécie de laboratório experimental que possibilita representar um sistema real por meio de um modelo. Esta permite determinar o comportamento do sistema quando algumas variáveis são modificadas (FITZSIMMONS E FITZSIMMONS, 2010). A despeito do fato desta técnica ser conhecida há algum tempo, apenas recentemente, foram desenvolvidos aplicativos que permitem a construção de modelos de simulação por não-especialistas. Alguns destes são dedicados às sistemas de serviços (CORRÊA E CAON, 2002).

O estudo de tempos é uma técnica utilizada no desenvolvimento de padrões de desempenho. É preciso identificar e medir o tempo gasto pelas atividades que compõem os processos produtivos repetitivos. Essas medições podem ser feitas com cronômetro. O tempo gasto por cada tarefa deve ser registrado e analisado estatisticamente para ser empregado como uma referencia no dimensionamento do 
tempo consumido pelo processo. Estes dados podem ser também utilizados para fins de planejamento e orçamento dos custos, balanceamento das operações, melhorias de desempenho, avaliação individual de trabalhadores, servir de base para pagamento de salários, podendo ainda serem utilizados na definição de padrões para a produtividade da mão de obra. (FITZSIMMONS E FITZSIMMONS, 2010, SCHMENNER, 1999).

Lovelock, Wirtz e Hemzo (2011) reconhecem que o tempo desempenha papel fundamental quando se trata de serviços, tanto para as suas operações quanto para os clientes. É importante, deste modo, planejar a sequência na qual os clientes utilizarão os serviços principais e suplementares e o tempo requerido em cada situação.

A Figura 2 mostra os métodos e técnicas referenciados nesta seção e a fonte bibliográfica que contém tal método.

Figura 2 - Relação de métodos e técnicas para o Projeto do Processo

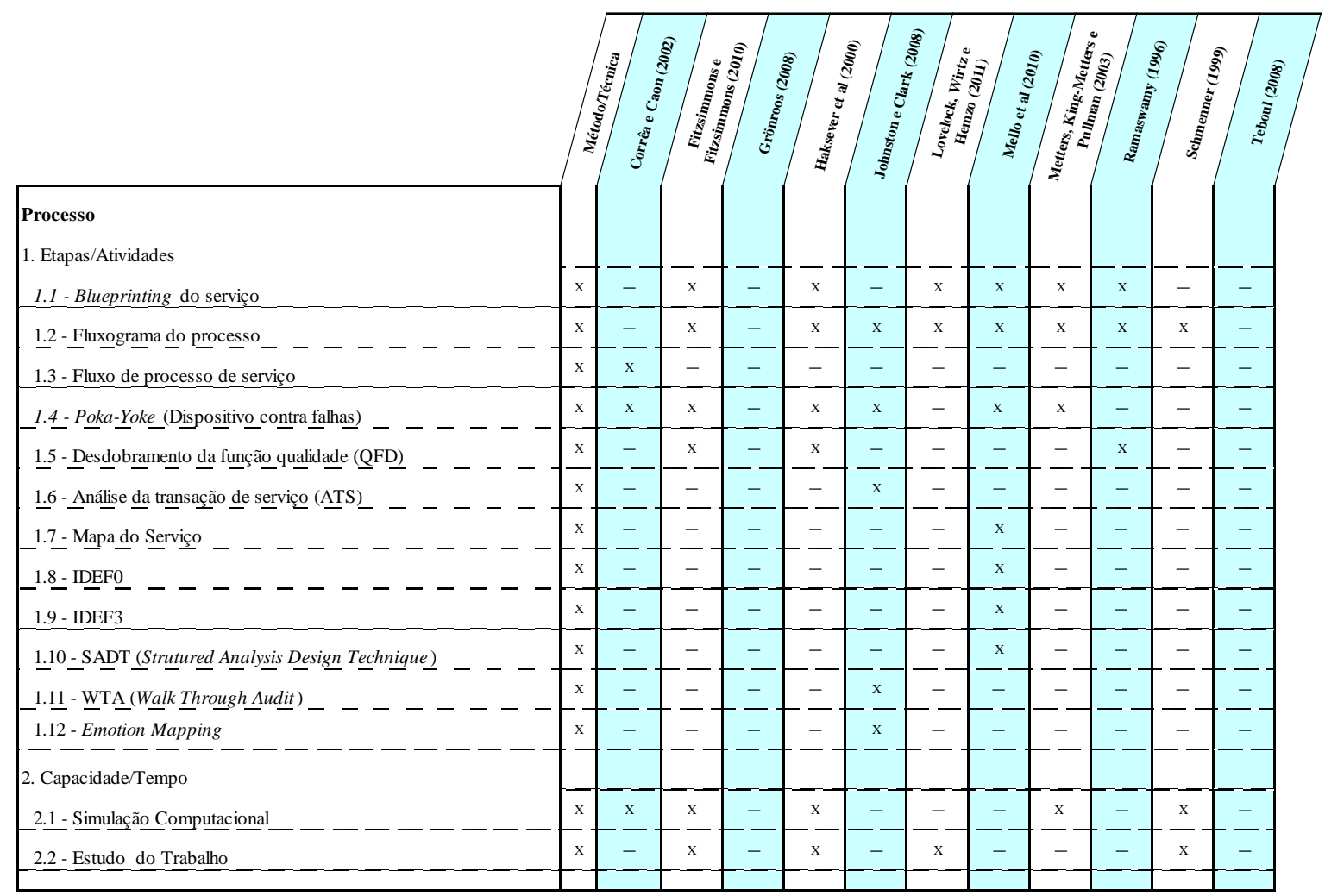

Fonte: Pesquisa dos Autores (2012). 


\subsection{Sistema do Serviço}

\section{Recursos Técnicos Físicos - Localização}

Diversos autores pesquisados citaram algumas técnicas relacionadas à definição da localização geográfica da instalação física do serviço.

Decisões relacionadas à localização são geralmente caras e podem impactar significativamente nos investimentos, custos operacionais e faturamento da empresa. Devem ser tomadas de maneira cuidadosa tendo-se como referência os fatores existentes relacionadas à capacidade produtiva e demanda pelos serviços (JOHNSTON E CLARK, 2008).

A técnica da representação geográfica dispõe as opções de localização e as distâncias de percurso na forma de rede. Posteriormente, as distâncias entre os diversos pares de opções são calculadas utilizando-se da distância euclidiana ou metropolitana. Dados relacionados aos problemas ambientais devem ser utilizados para a escolha da melhor opção (FITZSIMMONS E FITZSIMMONS, 2010).

Critérios de otimização podem definir a localização ideal por meio de objetivos distintos para o setor público e para o setor privado, uma vez que os interesses de cada um podem ser diferentes. O setor privado normalmente considera o equilíbrio entre os custos de construção e operação das instalações e custos de transporte. O setor público leva em conta a distância média percorrida para chegar ao local, a capacidade de geração de demanda e o orçamento disponível. Para o setor privado, o objetivo principal será o de minimizar os custos, ou aumentar os ganhos. Já para o setor público, o objetivo principal será maximizar um benefício social (FITZSIMMONS E FITZSIMMONS, 2010).

Um método qualitativo e quantitativo para a seleção da localização do negócio é o método da ponderação dos fatores locacionais. Este é bastante simples e pode ser resumido em seis passos: i) criar uma lista dos fatores locacionais relevantes; ii) designar um peso a cada fator; iii) criar uma escala para cada fator; iv) avaliar cada fator, atribuindo uma nota, conforme a escala criada; v) para cada fator, multiplicar a nota obtida pelo peso designado, e somar as notas obtidas pelos fatores de cada localização avaliada; e vi) escolher a localização que apresentar o melhor resultado, considerando também eventuais critérios qualitativos (CORRÊA e CAON, 2002). 
Para Metters, King-Metters e Pullman (2003) a classificação de fatores define alguns critérios chaves e a cada um deles se fixa pesos. Para cada alternativa de local e a cada critério são atribuídos notas. A combinação dos pesos dos critérios e das notas obtidas compõem o valor total que definirá a melhor localização. Portanto, o local com maior pontuação é o mais indicado.

A regressão linear também pode ser utilizada como um método de seleção de localização para os serviços. Esta é similar à classificação de fatores, a diferença é que os pesos dos fatores são determinados pela equação de regressão (METTERS; KING-METTERS E PULLMAN, 2003).

O sistema de informação geográfica é um software computacional que vincula a localização e outras informações importantes para o negócio em um formato visual de simples utilização. O sistema prevê a demanda baseado em informações armazenadas em bases de dados geográficas que definirão o melhor local (METTERS; KING-METTERS E PULLMAN, 2003).

Haksever et al. (2000) apresenta o método dos fatores dominantes de localização. Este é baseado em nove fatores relevantes relacionados à localização. São eles: os fatores baseados no cliente, baseados nos custos, baseados nos concorrentes, os sistemas de suporte, os fatores geográficos ou ambientais, o clima do negócio, os fatores baseados em comunicação, os baseados em transporte e por fim os principais desejos pessoais do CEO da empresa. Além dos fatores dominantes há outros critérios gerais que devem ser considerados na seleção de localização, tais como: disponibilidade de força de trabalho e o seu custo, o clima da região, os impostos cobrados pelo local, o transporte e a rede rodoviária, entre outros.

Quanto ao número de instalações, quando se opta por uma instalação única, não há muita dificuldade na definição de seu local. Utiliza-se a técnica da métrica metropolitana ou euclidiana, que pretende localizar uma instalação única sobre um plano, de forma a minimizar as distâncias percorridas (FITZSIMMONS E FITZSIMMONS, 2010).

O método centro de gravidade é uma abordagem para solucionar o problema da localização única, por meio do uso de fórmulas que possibilitam uma interpretação física das funções de deslocamento. (FITZSIMMONS E FITZSIMMONS, 2010). 
A localização de lojas consiste na utilização de um modelo de gravidade para se estimar a demanda dos consumidores. Este segue um procedimento exaustivo para calcular o lucro anual esperado de cada potencial localização em vários tamanhos de loja em um dado local. Objetiva maximizar o lucro por meio da avaliação de um número distinto de alternativas (FITZSIMMONS E FITZSIMMONS, 2010).

Quando se opta por múltiplas instalações, os cálculos são mais complexos e envolvem mais variáveis, o que por vezes não gera resultados ótimos. O problema da definição da área de cobertura da localização tenta encontrar o número mínimo de localizações e os locais das mesmas que servirão a todos os pontos de demanda dentro de uma distância máxima de serviços especificada. Há também o problema da definição da localização com a maior cobertura, em que se acrescenta a uma rede de percurso várias informações sobre a população usuária em cada comunidade. Busca-se maximizar a população atendida dentro de uma área de serviço desejada (FITZSIMMONS E FITZSIMMONS, 2010). Este último problema é também conhecido como método de cobertura máxima e é aplicado a alguns serviços em áreas metropolitanas que requerem múltiplos postos de atendimento. Os critérios utilizados para definição da localização e o tamanho desses postos de atendimento são: distância, tempo e custo (CORRÊA E CAON, 2002).

A técnica filiais versus operação única de serviço está baseada nas muitas semelhanças entre as operações com filiais próprias e as operações únicas, seja no tamanho, no tempo que operam naquele mesmo local, no tipo de espaço que ocupam, nas relações capital/mão de obra ou no grau de interação com os clientes. Em termos estatísticos, filiais são diferentes de operações únicas, pois elas valorizam mais a proximidade com o cliente e oferecem um serviço mais padronizado. Sua localização costuma ter por objetivo a proximidade a áreas com maior densidade demográfica e tráfego intenso, além disso, buscam neutralizar a influência de seus concorrentes (SCHMENNER, 1999).

Outra opção para as organizações de serviços é criar fábricas de serviço em uma escala bem pequena para maximizar a cobertura em determinada área geográfica, são as chamadas minilojas. Um exemplo destas instalações são os quiosques localizados nos shoppings centers. É possível ainda a localização de organizações de serviço em instalações multipropósito, em que são instalados 
serviços de varejo e outros tipos em rotas de transporte ou mesmo em terminais de ônibus, trens ou aéreos, por exemplo. As empresas necessitam verificar ainda as limitações de localização, pois em algumas situações é mais conveniente que o serviço vá até o cliente e não o contrário ou há restrições geográficas que impedem determinada localização (LOVELOCK, WIRTZ, HEMZO, 2011).

\section{Recursos Técnicos Físicos - Arranjo Físico e Servicescape}

Os autores pesquisados discutem os três principais tipos de layout: por produto, processo e posicional. O layout por produto se adequa a serviços padronizados divididos em uma sequência de etapas, pelas quais todo cliente deve passar (FITZSIMMONS EFITZSIMMONS, 2010). Este tipo de layout é necessário em processos cujo serviço seja produzido de forma repetitiva, uniforme e em grandes quantidades. Os recursos são arranjados de forma a favorecer o fluxo rápido e eficiente de clientes (CORRÊA E CAON, 2002).

O layout por processo permite ao cliente decidir a sequência de atividades e serviços que ele irá realizar, indo ao encontro de suas necessidades, possibilitando certo grau de customização. O arranjo físico é planejado com o objetivo de minimizar as distâncias percorridas ou o fluxo entre um departamento e outro (FITZSIMMONS E FITZSIMMONS, 2010).

No arranjo físico posicional, o cliente, em vez de se deslocar entre os recursos como nos dois arranjos anteriores, fica estacionário em uma posição definida. Os recursos, então, vão até ele, o que permite maior grau de contato com o cliente e maior personalização do serviço (CORRÊA E CAON, 2002).

Para Metters, King-Metters e Pullman (2003) a escolha do layout das instalações de serviço pode ser visto como dependente da presença do cliente. Quando o cliente não está presente, como em atividades de retaguarda, as decisões de layout devem ser abalizadas principalmente pela busca de eficiência operacional. Mas quando o cliente está fisicamente presente, as decisões de layout giram em torno de como o ambiente físico pode afetar adequadamente sua percepção acerca da qualidade do serviço.

Segundo Haksever et al (2000) os problemas de layout envolvem encontrar o melhor arranjo para os componentes físicos do sistema de serviço dentro das 
restrições de tempo, custos e tecnologia. Os autores mostram a técnica OPQRST. Esta consiste na discussão de seis questões antes de se tomar a decisão de layout: objetivos da organização, pessoas/serviços, quantidade de outputs demandados, roteamento, espaço disponível, serviços oferecidos e o tempo do processo.

Alguns autores mostram a importância da evidência física para comunicar os atributos de qualidade dos serviços. As evidencias auxiliam na definição das expectativas dos clientes e contribuem significativamente para criação da experiência nos serviços. Neste sentido, todos os aspectos de instalações físicas da organização bem como outras formas de comunicação tangível atingem os clientes e informam algo acerca do serviço aos mesmos, além de facilitarem o desempenho do serviço. Pesquisadores de comportamento do consumidor argumentam que as evidências físicas podem influenciar as escolhas dos clientes, suas expectativas, sua satisfação e outros comportamentos. Portanto, as evidências físicas podem ser entendidas de modo mais amplo como se formassem um cenário de serviços ou "Servicescape" (ZEITHAML, BITNER e GREMLER, 2011; BITNER, 1992; LOVELOCK, WIRTZ, HEMZO, 2011). Dada sua importância, os autores Fitzsimmons e Fitzsimmons (2010) argumentam que o "Servicescape" seja projetado de maneira coerente com o conceito do serviço a ser entregue.

\section{Sistema do Serviço - Sistemas de Controle}

Baseando-se no trabalho de Simons, os autores Johnston e Clark (2008) colocam que há quatro sistemas de controle que orientam o gerente de serviço a delegar maior autonomia aos funcionários: i) sistema denominado crença, que objetiva a cooperação entre os funcionários e depende de uma cultura organizacional bem articulada; ii) sistema de limites, que tem o intuito de delimitar a iniciativa dos empregados, para se obter obediência e evitar riscos; iii) sistemas de diagnóstico que definem metas a serem atingidas pelos colaboradores da empresa; iv) sistema de controle interativo, que estimula a criação, e é mais adequado às "indústrias de conhecimento", uma vez que tais organizações devem oferecer soluções criativas a seus clientes. Para Schmenner (1999) os sistemas de controle são mais importantes para o setor de serviços do que para o setor industrial, uma vez que estes apresentam uma série de características que demandam maior 
controle por parte da gerência. São exemplos: os serviços não acumulam estoques e, normalmente, a demanda é errática, sendo comum a existência de filas; os clientes podem interferir no processo; há menos variáveis mensuráveis relativas à qualidade de produto, o que exige mais atenção à prevenção de falhas.

Para Grönroos (2009), o foco do controle e da supervisão no gerenciamento de serviços deve estar no incentivo e apoio aos colaboradores, uma vez que nem sempre os serviços podem ser totalmente padronizados. Desse modo, é necessária maior flexibilidade para que haja qualidade e atendimento das expectativas dos clientes.

Outra abordagem que trata do controle versus envolvimento dos funcionários nos serviços enfatiza que quatro dimensões são fundamentais para a escolha da abordagem correta. São elas: i) informação sobre o desempenho organizacional; ii) existência de conhecimento sobre o processo pelos funcionários que os habilitem a entender e contribuir para este desempenho seja alcançado; iii) poder de tomar decisões; iv) sistema de recompensas baseadas no desempenho organizacional (LOVELOCK, WIRTZ, HEMZO; 2011).

\section{Sistema do Serviço - Funcionários}

Dada sua importância para a satisfação do cliente, os autores Fitzsimmons e Fitzsimmons (2010) sugerem algumas práticas para a avaliação dos funcionários de linha de frente durante o processo seletivo pela empresa. A seleção pode incluir avaliações que podem ser feitas de três maneiras: o questionamento abstrato consiste em uma entrevista baseada em questões abertas; o esboço situacional requer que o entrevistado responda a questões relativas a uma situação específica; e o desempenho de um papel requer que os entrevistados participem de uma situação simulada e reajam como se o ambiente fosse real.

O treinamento dos funcionários deve considerar que as dificuldades nas interações entre os clientes e o pessoal da linha de frente podem ser ocasionadas por dois motivos principais: expectativas não realistas dos clientes em relação ao serviço e falhas inesperadas do mesmo. Desse modo, os funcionários devem ser treinados para evitarem que uma situação ruim fique ainda pior, e também devem 
adquirir habilidades de comunicação para lidar com estas situações (FITZSIMMONS E FITZSIMMONS, 2010).

Para Grönroos (2009) o treinamento dos funcionários deveria incluir o desenvolvimento de uma visão mais holística da estratégia de serviço e do processo total existente de marketing, além de incentivar atitudes favoráveis em relação à estratégia do serviço e ao foco no cliente, devendo ainda aprimorar as capacidades de comunicação, vendas e serviços entre os empregados da organização.

Corrêa e Caon (2002) afirmam que os funcionários, além das habilidades técnicas requeridas para a prestação de serviços, sejam, em determinadas situações, atraentes, transmitam tranquilidade, segurança e administrem as relações entre o cliente e o eventual elemento de tecnologia envolvido. O grau necessário de habilidades interpessoais vai variar conforme a intensidade de contato que a operação exige. O funcionário deve ter habilidades de comunicação, pois uma correta formação de expectativas e percepções no cliente depende da comunicação adequada entre os funcionários e a empresa. Funcionários de contato normalmente necessitam ter habilidades para vendas.

Os principais requisitos gerais dos funcionários em uma organização focada no cliente e orientada para os serviços são entender o relacionamento total que a empresa tem com seus clientes, compreender e aceitar seu papel na manutenção destes relacionamentos, e ter um comportamento focado no cliente dentro do ambiente de trabalho. Os funcionários também devem ter habilidades para interagir e se comunicar com os clientes e devem se sentir motivados para agirem deste modo (GRONROOS, 2009).

A programação de mão de obra nas empresas de serviços abrange duas questões principais. A primeira diz respeito à quantidade de funcionários que deve estar trabalhando em qualquer momento. Quando a demanda e a produtividade da força de trabalho forem conhecidas, a quantidade de funcionários é relativamente fácil de ser estimada e essa questão pode ser tratada utilizando a Programação Linear. Na existência de incertezas significativas com relação a estes dois aspectos, a organização deve estabelecer certo excesso de capacidade. A segunda questão refere-se à definição dos turnos de trabalho. Isto envolve a escolha inteligente dos diferentes tipos de turnos em que os trabalhadores de serviços frequentemente 
possam ser escalados e depois a execução de alguma simulação para ver qual a influência das escolhas feitas sobre os custos (SCHNENNER, 1999).

Teboul (2008) propõe algumas alternativas para contornar o problema da falta de pessoal nas organizações. A primeira delas é programar os turnos de trabalho de acordo com a previsão da demanda. A segunda alternativa é utilizar colaboradores em meio expediente para complementar o quadro permanente ou realizar subcontratações. É possível também realizar um compartilhamento de pessoal entre os departamentos da empresa. Por fim, é sugerido pelo autor que se tenha colaboradores polivalentes, o que favorece o remanejamento de funcionários nos momentos em que for necessário.

\section{Sistema do Serviço - Clientes}

O cliente pode ser visto como um funcionário parcial, uma vez que ele é uma parte integral do sistema de prestação de serviço e pode desempenhar vários papéis, dependendo da natureza da atividade e da abordagem determinada pelo serviço. Ele pode ser um especificador do serviço, concedendo informações claras sobre as exigências, antes do produto-serviço apropriado ser selecionado e entregue. O cliente pode ter um papel de inspetor de qualidade, o que pode ser obtido por meio de mecanismos formais de feedback, como grupos de foco ou questionários. O cliente pode ainda atuar como co-produtor, sendo parte da atmosfera para outros clientes ou um recurso para a organização em situações de auto-serviço. O envolvimento do cliente no processo de prestação do serviço frequentemente faz com que ele desenvolva maior senso de lealdade e reduz a necessidade de recursos próprios. Se ele executar algumas tarefas, essas possibilitarão certa sensação de maior controle pessoal sobre o que acontece para ele (BOWEN, 1986; JOHNSTON E CLARK, 2008).

Para Teboul (2008) outro benefício da aplicação da participação do cliente na prestação do serviço é a redução de custos e também do tempo de espera. Esta estratégia é amplamente utilizada por grandes redes de fast-food e de demais serviços de massa.

Os clientes podem ser também entendidos como um recurso no processo de marketing interativo, na medida em que podem ser coprodutores do serviço. 
Entretanto, a disposição deles para a coprodução não é automática e, sempre que necessário, eles tem de ser educados para cooperarem de modo correto no processo do serviço e ainda para que sejam capazes de perceber a qualidade do serviço (GRONROOS, 2009).

É possível atribuir uma tipologia da participação dos clientes na produção do serviço. Em um extremo, no nível baixo de participação, os funcionários e sistemas realizam todo o trabalho e são poucos os momentos de contato com o cliente. No nível moderado de participação, são requeridos insumos para ajudar a organização a criar e entregar o serviço com um certo grau de customização. É importante a comunicação educativa para que a coprodução resulte em um serviço satisfatório. Já em outro extremo, no nível alto de participação, os clientes desenvolvem um trabalho ativo com o fornecedor para coproduzir o serviço. Este não pode ser entregue sem uma grande participação do cliente (LOVELOCK, WIRTZ, HEMZO; 2011).

A Figura 3 a seguir mostra os métodos e técnicas identificados nesta seção, bem como a fonte bibliográfica utilizada. 
Figura 3 - Relação de métodos e técnicas para o Projeto do Processo

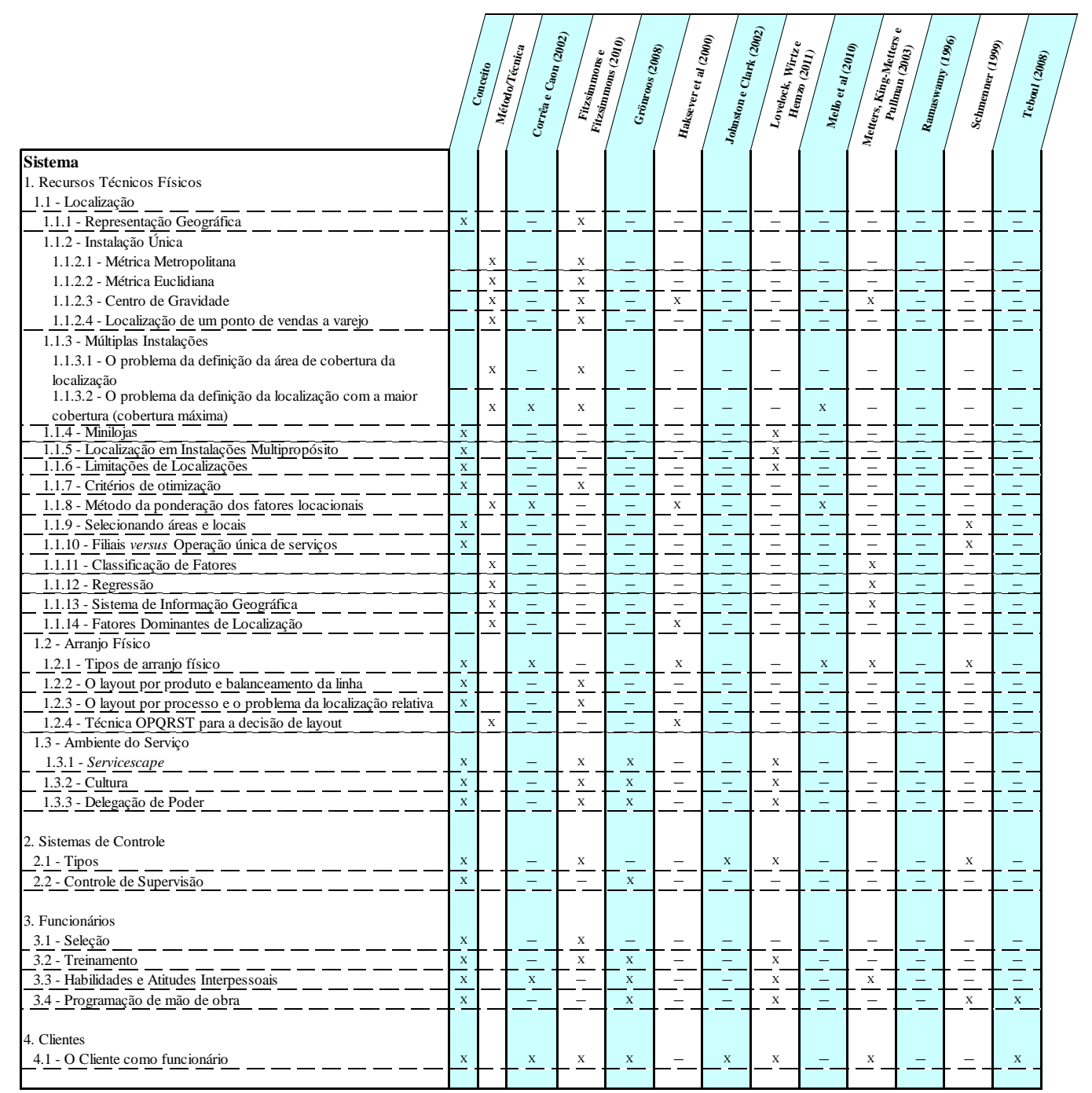

Fonte: Pesquisa dos Autores (2012).

\section{CONSIDERAÇÕES FINAIS}

As Figuras 1, 2 e 3 reportam os diferentes métodos e técnicas utilizados para o projeto de serviços encontrados na literatura pesquisada. Uma análise destas Figuras revela que o conhecimento acerca dos métodos e técnicas ainda está difuso entre os autores. Pode-se observar também que o projeto do processo e a definição da localização dos serviços dispõem de um conjunto maior de métodos e técnicas. Entretanto, elementos do projeto de serviço como conceito do produto, arranjo físico, atmosfera do serviço, sistemas de controle e projeto de trabalho dos funcionários não estão com seu projeto apoiados em métodos e técnicas. Para estes elementos, os textos consultados estabelecem regras e princípios que guiam a concepção destes itens. Essas se situam num nível mais conceitual do que normativo e 
descritivo. Duas causas podem ser identificadas para a existência de poucos métodos e técnicas em detrimento da predominância de conceitos para o projeto de serviços. A primeira delas é o baixo nível de desenvolvimento deste tema na literatura (MENOR, TATIKONDA e SAMPSON, 2002). A segunda refere-se ao próprio setor de serviços que apresenta uma enorme quantidade de tipos e formatos de empresas. Essa grande diversidade dificulta enormemente a definição precisa de métodos e técnicas de projeto.

Os autores entendem que as informações construídas neste trabalho poderão ser úteis para os gestores, pesquisadores e estudantes da área de gerenciamento de serviços, uma vez que este trabalho poderá ser utilizado como um guia inicial para a escolha do método e técnica numa organização que esteja estruturando o Projeto de seus Serviços.

Importante frisar que o trabalho também poderá ser útil para as organizações em funcionamento que tencionam reformular seus serviços. Os autores Berry e Lampo (2000) propõem cinco estratégias genéricas de redesenho. Cada uma destas estratégias poderá utilizar alguns dos métodos e técnicas sugeridos neste trabalho. Nesta direção, o Quadro 2 apresenta cada uma delas e aponta quais os principais elementos de projeto que deverão ser desenvolvidos na mesma.

O conhecimento acerca dos métodos e técnicas para o projeto de serviços pode ser entendido como relevante para à área de Engenharia de Produção. Haja visto que a análise de dois importantes periódicos nacionais desta área revelou a existência de vários trabalhos acerca da aplicação de métodos e técnicas de projeto, tais como: Modelagem, QFD, SFMEA, FTA, FMEA, SERVPRO, etc. Isto aponta que o tema analisado no trabalho é objeto de estudo e pesquisa pela comunidade acadêmica desta área. (DIAS JUNIOR, 2012; CRUZ JUNIOR e CARVALHO, 2003; FABRI e CARVALHO, 2005; OLIVEIRA, PAIVA e ALMEIDA, 2010; ROTONDARO, 2002; SANTOS e VARVAKIS, 2002; SILVA e RIBEIRO, 2002).

Metters (2010) destaca que cerca de $10 \%$ da pesquisa na área de gestão de operações está diretamente relacionada com o setor de serviços. O autor levanta alguns motivos para a ocorrência deste baixo percentual, e aponta algumas alternativas para modificar este quadro. Uma delas consiste na divulgação de trabalhos desta área no meio acadêmico, por meio do ensino e da pesquisa. Neste contexto, o presente texto busca disseminar os conhecimentos acerca do projeto de 
serviços existentes na literatura de operações para a comunidade de Engenharia de Produção.

Quadro 2 - Estratégias genéricas de redesenho e os principais elementos de projeto que deverão ser desenvolvidos

\begin{tabular}{|l|l|l|}
\hline \multicolumn{1}{|c|}{ Estratégia } & \multicolumn{1}{|c|}{ Benefícios } & \multicolumn{1}{|c|}{$\begin{array}{l}\text { Principais elementos } \\
\text { de Projeto trabalhados }\end{array}$} \\
\hline $\begin{array}{l}\text { Auto-serviço - colocar o } \\
\text { cliente no papel de produtor. }\end{array}$ & $\begin{array}{l}\text { Aumenta a percepção de controle pelo } \\
\text { cliente, aumenta a velocidade do serviço, } \\
\text { melhora o acesso e diminui os custos. }\end{array}$ & $\begin{array}{l}\text { - Processo } \\
\text { - Sistema de Controle } \\
\text { - Clientes }\end{array}$ \\
\hline $\begin{array}{l}\text { Serviço direto - serviço é } \\
\text { entregue no local do cliente }\end{array}$ & $\begin{array}{l}\text { Aumenta a conveniência, melhora o } \\
\text { acesso. }\end{array}$ & $\begin{array}{l}\text { - Processo } \\
\text { - Recursos Técnicos } \\
\text { - Empregados }\end{array}$ \\
\hline $\begin{array}{l}\text { Pré-serviço - agilizar um } \\
\text { conjunto de atividades } \\
\text { necessárias para a ativação } \\
\text { do serviço }\end{array}$ & $\begin{array}{l}\text { Melhora a velocidade e a eficiência do } \\
\text { serviço, redireciona tarefas do cliente para } \\
\text { o prestador, separa a ativação do serviço } \\
\text { da sua entrega, customiza o serviço. }\end{array}$ & $\begin{array}{l}\text { - Processo } \\
\text { - Sistema de Controle }\end{array}$ \\
\hline $\begin{array}{l}\text { Serviços Combinados - Emados } \\
\text { combina múltiplos serviços } \\
\text { em um único pacote }\end{array}$ & $\begin{array}{l}\text { Melhora a conveniência e customiza o } \\
\text { serviço. }\end{array}$ & $\begin{array}{l}\text { - Produto } \\
\text { - Processo } \\
\text { - Sistema de Controle } \\
\text { - Empregados }\end{array}$ \\
\hline $\begin{array}{l}\text { Evidência física do serviço - } \\
\text { manipulação dos elementos } \\
\text { tangíveis associados ao } \\
\text { serviço }\end{array}$ & $\begin{array}{l}\text { Melhora conveniência e o valor percebido } \\
\text { do serviço. }\end{array}$ & \begin{tabular}{l} 
- Recursos técnicos \\
\hline
\end{tabular} \\
\hline
\end{tabular}

Fonte: Berry e Lampo (2000).

O trabalho abordou apenas o projeto de serviços sob a ótica da Gestão de Operações, entretanto, o projeto de serviços pode ser compreendido por uma abordagem multidisciplinar nos quais áreas como Design de Produto, Design Gráfico, Sociologia, Etnografia, Marketing e Estratégia também se fazem presentes no desenvolvimento dos Serviços (STICKDORN e SCHNEIDER, 2011). Nesta direção, o desenvolvimento de serviços deve ser compreendido dentro de uma perspectiva maior denominada de "Service Science". Esta pode ser entendida como uma abordagem transdisciplinar para estudar, melhorar, criar e inovar os serviços, tendo como referência os paradigmas de co-criação de valor e sistema de serviços (SPOHRER e MAGLIO, 2010). Entretanto, para que esta abordagem interdisciplinar ocorra faz-se necessário um grande esforço de formação e pesquisa dentro das universidades e demais centros de pesquisa. Estes devem comtemplar à formação de alunos de graduação com o perfil e conhecimento nas diversas áreas que compõem a "Service Science" (BITNER e BROWN, 2008). 
O trabalho pode ser entendido como um estudo inicial sobre este tema, portanto, seria de grande valia uma pesquisa de campo em empresas de serviços tidas como inovadoras e competitivas no seu setor de atuação acerca do uso de métodos e técnicas para o projeto de serviços.

\section{REFERÊNCIAS}

BERRY, L. L.; S. K. LAMPO. Teaching an old service new tricks, Journal of Service Research, v. 2, n. 3, p. 265-75, 2000.

BITNER, M. J.. "Servicescape: the impact of physical surroundings on customers and employees", Journal of Marketing, v. 56, p. 57-71, April, 1992.

BITNER, M. J.; BROWN, S. W.. The service imperative, Business Horizons, v. 51, p. 39-46, 2008.

BOWEN, D. E.. Managing customers as human resources in service organizations. Human Resource Management, v. 25, n. 3, p. 371-383, 1986.

CHASE, R. B.. Revisiting "Where does the customer fit in a service operation?": Background and Future Development of Contact Theory. In. MAGLIO, P. P.; KIELISZEWSKI, C. A.; SPOHRER, J. C. (Editors). Handbook of Service Science, Springer Science+Business Media, p. 11-17, 2010.

CHASE, R. B.. Where does the customer fit in a service operation?, Harvard Business Review, Nov./Dec., p. 137-42, 1978.

CHASE, R.B.; STEWART, D. M.. Make Your Service Fail-Safe. Sloan Management Review, v. 35, n. 3, p. 35-44, 1994.

CHESBROUGH, H.; DAVIES, A.. Advancing Services Innovation: five key concepts. In. MAGLIO, P. P.; KIELISZEWSKI, C. A.; SPOHRER, J. C. (Editors). Handbook of Service Science, Springer Science+Business Media, p. 579-601, 2010.

CLARK, G.; JOHNSTON R.; SHULVER, M.. Exploiting the service concept for service design and development. In: FITZSIMMONS, J.A.; FITZSIMMONS, M.J. (Eds.) New Service Development - Creating Memorable Experiences. Thousand Oaks, CA: Sage Publications, p. 71-9, 2000.

CLARK, G.; JOHNSTON, R.; SHULVER, M.. Exploiting the service concept for service design and development. In. FITZSIMMONS, J.A.; FITZSIMMONS, M.J. (Eds.) New Service Development - Creating Memorable Experiences. Thousand Oaks, CA: Sage Publications, p. 71-91, 2000.

CORRÊA, H. L.; CAON, M.. Gestão de serviços: lucratividade por meio de operações e de satisfação dos clientes. São Paulo: Atlas, 2002. 
CRUZ JUNIOR, A.T.; CARVALHO, M. M. Obtenção da voz do consumidor: estudo de caso em um hotel ecológico. Prod., v.13, n. 3, p.88-100, 2003.

DIAS JUNIOR, F. J. et al. Desdobramento da função qualidade (QFD) no desenvolvimento de projeto de treinamento: estudo exploratório para serviço, Produção Online, v. 12, n. 1, 2012.

EDVARDSSON, B.. Quality in new service development: key concepts and a frame of reference. International Journal of Production Economics. v. 52, p. 31-46, 1997.

FABRI, J. A.; CARVALHO, M. M. QFD estendido em ambiente de gerenciamento de informações para ensino distância, Produção Online, v. 5, n. 2, 2005.

FISHER, C.; SCHUTTA, J. T.. Development New Services: incorporating the voice of the customer into strategic service development. Milwaukee: ASQ Press, 2003.

FITZSIMMONS, J. A.; FITZSIMMONS, M. J.. Administração de Serviços: operações, estratégia e tecnologia de informação. Porto Alegre: Bookman, 6.ed., 2010.

FITZSIMMONS, J.A.; FITZSIMMONS, M.J. (Eds.) New service development: creating memorable experiences. Thousand Oaks, CA: Sage Publications, 2000.

GIL, A. C.. Métodos e técnicas de pesquisa social. 3. ed. São Paulo: Atlas, 1991.

GRÖNROOS, C. Marketing: gerenciamento e serviços, Rio de Janeiro: Elsevier, 2008.

HAKSEVER, C. et al.. Service Management and Operations. Prentice Hall, 2000.

JOHNSTON, R.. Service transaction analysis: assessing and improving the customer's experience, Managing Service Quality, v. 9, n. 2, p. 102-109, 1999.

JOHNSTON, R.; CLARK, G.. Service Operations Management: improving service delivery, Harlow: Pearson Education Limited, $3^{\circ}$ Edition, 2008.

KINGMAN-BRUNDAGE, J.; GEORG, W.R.; BOWEN, D .E.. Service logic: achieving service system integration. International Journal of Service Industry Management, v. 2, n. 3, p. 20-39, 1995.

LEVITT, T.. The industrialization of service. Harvard Business Review, p. 63-75, Set./Out., 1976.

LOVELOCK, C.; WIRTZ, J.; HEMZO, M. A., Marketing de Serviços: pessoas, tecnologia e estratégia. São Paulo: Pearson Prentice Hall, 7. ed. 2011.

MACHUCA, J. A.D.; GONZÁLEZ-ZAMORA, M. del M; AGUILAR-ESCOBAR, V. G.. 
Service operations management research. Journal of Operations Management. v.25, p. 585-603, 2007.

MELLO, C. H. P. et al.. Gestão do processo de desenvolvimento de serviços. São Paulo: Atlas, 2010.

MENOR, L.J.; TATIKONDA, M.V.; SAMPSON, S.E.. New service development: areas for exploitation and exploration. Journal of Operations Management, v. 20, p. 135-157, 2002.

METTERS, R.; KING-MATTERS, K; PULLMAN, M;. Successful service operations management. South-Western College Pub, 2003.

METTERS, R. The Neglect of service science in the operations management field, In. MAGLIO, P. P.; KIELISZEWSKI, C. A.; SPOHRER, J. C. (Editors). Handbook of Service Science, Springer Science+Business Media, 2010. p. 309-319.

OLIVA, R.; KALLENBERG, R.. Managing the transition from products to services, International Journal of Service Industry Management, v. 14, n. 2, 2003.

OLIVEIRA, U. R.; PAIVA, E. J.; ALMEIDA, D. A. Metodologia integrada para mapeamento de falhas: uma proposta de utilização conjunta do mapeamento de processos com as técnicas FTA, FMEA e a análise crítica de especialistas. Prod., mar, v. 20, n.1, p.77-9, 2010.

RAMASWAMY, R.. Design and management of service process: keeping customer for life. Prentice Hall, 1996.

ROTONDARO, R. G.. SFMEA: análise do efeito e modo da falha em serviços aplicando técnicas de prevenção na melhoria de serviços. Prod., v.12, n. 2, p.54-62, 2002.

ROZENFELD, H. et al.. Gestão de desenvolvimento de produtos: uma referência para a melhoria do Processo, São Paulo: Editora Saraiva, 2006.

SANTOS, L. C.; VARVAKIS, G. SERVPRO: uma técnica para a gestão de operações de serviços. Prod., v.12, n.1, p.34-45, 2002.

SANTOS, L. C.; VARVASKIS, G.. Projeto e análise de processos de serviços: uma avaliação de técnicas de representação. Produto \& Produção, v. 5, n.3, p. 01-16, out. 2001.

SCHMENNER, R. W.. Administração de operações em serviços. São Paulo: Futura, 1999.

SHOSTACK, L.. Designing services that deliver, Harvard Business Review, Jan./Feb., p. 133-139, 1984.

SILVA, P. R. C.; RIBEIRO, J. L. D. Uma proposta para a modelagem do valor 
percebido na prestação de serviços. Prod., v.12, n.1, p.18-33, 2002.

SPOHER, J. C; MAGLIO, P. P. Toward a Science of Service Sytems: Value and Symbols. In. MAGLIO, P. P.; KIELISZEWSKI, C. A.; SPOHRER, J. C. (Editors).

Handbook of Service Science, Springer Science+Business Media, 2010, p. 11-17.

STICKDORN, M.; SCHNEIDER, J.. This is service design thinking: basics - tools - cases, Hoboken: John Wiley \& Sons, 2011.

TEBOUL, J.. Serviços em cena: o diferencial que agrega valor ao seu negócio. Brasília: IEL/NC, 2008.

ZEITHAML, V.A; BITNER, M.J.; GREMLER, D. D. Marketing de serviços: a empresa com foco no cliente. 5. ed. Porto Alegre: Bookman, 2011.

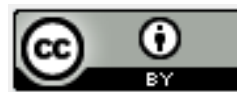

Artigo recebido em 20/08/2012 e aceito para publicação em 01/11/2012. 